\title{
Improvement of left ventricular systolic function in inflammatory cardiomyopathy: What plays a role?
}

\author{
Jan Krejcia, Petr Hude , Eva Ozabalovaa , Dalibor Mlejnek ${ }^{\mathrm{a}}$, Vita Zampachova ${ }^{\mathrm{b}}$, Iva Svobodova ${ }^{\mathrm{b}}$, Radka Stepanovac, \\ Lenka Spinarova ${ }^{a}$
}

\begin{abstract}
Aims. To compare the differences between patients with inflammatory cardiomyopathy (ICM) with and without improvement in left ventricular (LV) systolic function and to identify the relevant predictors of LV improvement.

Patients and Methods. The study included 63 patients with biopsy-proven ICM and heart failure symptoms of at least NYHA II, symptom duration $\leq 6$ months, LV ejection fraction (LVEF) $\leq 40 \%$ assessed by echocardiography and presence of $>14$ mononuclear leukocytes (LCA+ cells) $/ \mathrm{mm}^{2}$ in biopsy samples. Patients were evaluated at baseline and after 6 months.

Results. In the group with LVEF improvement of $\geq 10 \%$ (I+ group, $n=41)$, LVEF increased from $24 \pm 7 \%$ to $47 \pm 8 \%(P<$ 0.001 ). In 22 patients (group I-), there was no or minimal LVEF increase $(<10 \%)$. In the I+ group, there were more LCA+ cells $/ \mathrm{mm}^{2}$ at baseline $\left(25.1 \pm 16.5\right.$ vs. $18.5 \pm 4.4$ cells $\left./ \mathrm{mm}^{2} ; P=0.032\right)$ and a more significant decrease in LCA+ cells in the follow-up (reduction of $13.6 \pm 14.3$ cells $/ \mathrm{mm}^{2}$ vs. $5.0 \pm 7.7 \mathrm{cells} / \mathrm{mm}^{2}$ in the I- group; $P=0.009$ ). The univariate logistic regression showed a possible association of number of $L C A+$ cells, $L V$ end-diastolic diameter and $\mathrm{N}$-terminal fragment of pro-brain natriuretic peptide (NTproBNP) value with LVEF improvement. In the multivariate analysis, only NTproBNP at diagnosis was confirmed as an independent predictor of LVEF improvement ( $O R=1.2 ; 1.003$ to $1.394 ; P=0.046)$.

Conclusion. The LV systolic function improvement was observed in $65 \%$ of the patients. In these patients, the number of inflammatory cells at baseline was higher and decreased more but the higher baseline NTproBNP value was the only independent predictor of LVEF improvement.
\end{abstract}

Key words: inflammatory cardiomyopathy, endomyocardial biopsy, echocardiography, NTproBNP, right heart catheterization.

Received: April 16, 2016; Accepted: June 6, 2016; Available online: June 20, 2016

http://dx.doi.org/10.5507/bp.2016.033

${ }^{a}$ Department of Cardiovascular Diseases, St. Anne's University Hospital Brno and Masaryk University, Brno, Czech Republic

${ }^{b}$ First Department of Pathological Anatomy, St. Anne's University Hospital in Brno and Masaryk University, Brno, Czech Republic 'Department of Internal Medicine and Cardiology, University Hospital Brno, Czech Republic

Corresponding author: Jan Krejci, e-mail:jan.krejci@fnusa.cz

\section{INTRODUCTION}

Dilated cardiomyopathy (DCM) is the most common cause of end-stage heart failure in patients undergoing heart transplantation ${ }^{1}$. The causes of dilated cardiomyopathy (DCM) vary, but myocarditis is currently considered the most frequent cause ${ }^{2}$. The seriousness of this disease is underlined by the fact that it affects predominantly younger people in productive age. The most common manifestation of myocarditis is heart failure. If the myocarditis is associated with left ventricular systolic dysfunction, the disease is called inflammatory cardiomyopathy (ICM). Other clinical manifestations of myocarditis are chest pain and/or arrhythmias ${ }^{3-5}$. The cases presenting with heart failure symptoms have a poorer prognosis than those with chest pain or arrhythmias.

Clinical suspicion of myocarditis is raised by the presence of any of the described symptoms and simultaneous fulfillment of at least one of the diagnostic criteria from examinations such as ECG, laboratory findings, echocardiographic or magnetic resonance imaging as described elsewhere ${ }^{4}$. However, the definitive diagnosis of myocar- ditis is always histological and/or immunohistochemical which require a sample of myocardium for confirmation of the inflammation ${ }^{4,7-9}$.

A myocardial sample is commonly obtained transvascularly from the right or left ventricle using endomyocardial biopsy (EMB). EMB is considered the gold standard for myocarditis diagnosis and is also the only method able to confirm a final diagnosis in vivo ${ }^{6-10}$. According to the WHO / ISCF definition of 1995 diagnosis of myocarditis is based on histological, immunological and immunohistochemical criteria ${ }^{11}$.

Apart from the classical histological Dallas criteria which are highly specific but lack sensitivity ${ }^{12}$, immunohistochemical methods should also be used in the assessment of EMB samples (typing of infiltrating cells and/or grading of HLA antigen expression). These methods increase the diagnostic accuracy of the EMB but unfortunately the diagnostic criteria used in published studies are rather diverse and there is still no widely accepted consensus ${ }^{4,13-17}$.

The most common cause of heart muscle inflammation in Western civilizations is viral infection. Investigation of possible infectious agents has to be a part of the EMB 
assessment, most frequently by polymerase chain reaction (PCR) (ref. $\left.{ }^{4,7,9}\right)$. Non-viral infections as the cause of myocarditis are uncommon in our region, although in some endemic areas of the Czech Republic, Borrelia burgorferi is relatively often detected in recent onset dilated cardiomyopathy (RODCM) (ref. $\left.{ }^{18-20}\right)$. Other possible causes of myocarditis are autoimmune or toxic factors. Autoimmunity is a possible reaction to the initial viral damage of myocytes and is considered one of the main causes of LV function impairment in the second phase of the disease $\mathrm{e}^{10,21,22}$.

Immunohistochemical methods for EMB evaluation have shown that in $40-50 \%$ of DCM cases, myocarditis is present ${ }^{23,24}$. It has been reported that LV function improvement in ICM is greater than in DCM cases with no proven myocardial inflammation ${ }^{25}$. Improvement of LV function is associated with the retreat of inflammation. This is particularly evident in the case of fulminant forms of myocarditis with severe initial LV dysfunction; a significant improvement or even complete normalization of LV systolic function may occur in several weeks ${ }^{26}$. For this reason, the initial vigorous immune response may be a favorable factor in terms of long-term $\operatorname{prognosis}^{27}$.

However, the whole group of patients with biopsyproven myocarditis is not homogeneous in this regard. It is estimated that in about $50-70 \%$ of patients, the healing of myocarditis is associated with the improvement or normalization of LV systolic function. On the other hand, in some cases, LV dysfunction persists or even progresses to refractory failure ${ }^{4,5,7-10,28}$. There are a number of factors that play a role in the course of the disease, for instance type of myocarditis, grade of initial myocardial injury, hormonal factors, physical stress during the acute phase of the disease, the intensity and duration of the inflammatory process and persistence or replication of the virus in the myocardium ${ }^{5,7,9,10,28-30}$.

Although a number of studies have focused on prognostic indicators ${ }^{6,25,29-32}$, we have no clear and convincing evidence that will allow us to stratify patients at the time of diagnosis and to tailor additional medical care, including the use of advanced and expensive methods of current medicine, i.e. device therapy and heart transplantation. These considerations formed the motivation for this study.

The aim of our study was to compare the differences between a group of ICM patients with a clinically relevant improvement in LV function characterized by the rise in LVEF of at least $10 \%$, and a group where improvements in LVEF was smaller or entirely absent. We also tested for correlations between selected variables to identify possible predictors of improvement of LV systolic function.

\section{PATIENTS AND METHODS}

The study included 63 patients with biopsy-proven ICM, 46 men and 17 women, mean age $43 \pm 13$ years, who fulfilled inclusion criteria and had no exclusion ones. Inclusion criteria were as follows: heart failure symptoms of at least NYHA II, symptoms duration $\leq 6$ months, left ventricular ejection fraction (LVEF) $\leq 40 \%$ assessed by echocardiography, biopsy findings of $>14$ mononuclear leukocytes cells $/ \mathrm{mm}^{2}$ (LCA+ cells) consistent with the diagnosis of lymphocytic myocarditis (ref., $811,13,14,16,31,33$ ). Patients with coronary artery disease, significant valvular disease, toxic myocardial damage caused by alcohol or chemotherapy, endocrine disorders or tachycardiainduced LV dysfunction were excluded. Patients with specific forms of myocarditis (i.e. giant cell myocarditis or eosinophilic myocarditis) were excluded as well.

Patients were evaluated at the baseline visit (at the time of diagnosis) and after 6 months. Clinical examination, laboratory assessment, echocardiography and invasive evaluation including endomyocardial biopsy and right heart catheterization were performed at both visits. All enrolled patients were treated with standard heart failure therapy; immunosuppressive therapy was precluded in this study. All patients signed an informed consent prior to enrollment in the study; the study was approved by the institutional Ethics committee.

In the whole patients group, LVEF at the baseline was $25 \pm 8 \%$, the number of infiltrating LCA+ cells was $24.5 \pm$ $13.9 / \mathrm{mm}^{2}$, duration of symptoms at the time of diagnosis was $2.3 \pm 2.0$ months and functional class according to NYHA classification was $2.5 \pm 0.6$.

Patients were divided in two groups according to the presence of the LVEF improvement; the threshold was $10 \%$ increase in LVEF, the group with improvement was classified as I+, the group without improvement was called I-. Some other echocardiographic parameters, clinical status and selected hemodynamic and laboratory variables were compared for the two groups.

The EMB was performed with echocardiographic control under local anaesthesia from the right ventricle (RV) through the right internal jugular vein. Four samples were taken for histological and immunohistochemical evaluation and five samples for PCR analysis. In addition to standard histological assessment, the average number of T-lymphocytes (CD3+ cells) and mononuclear leukocytes $(\mathrm{LCA}+$ cells $)$ in myocardial interstitial spaces were determined. For the purpose of this study, only the number of LCA+ cells was decisive (see Fig. 1). LCA+ cells were stained with monoclonal antibody Anti-Human CD45/ LCA DakoCytomation. The numbers of infiltrating cells were evaluated in 4 sections performed on two glasses with a step sectioning. The final result was the average from $28 \pm 4$ thinnings of the samples.

Echocardiographic examinations were performed on Vivid E9 machine (GE, Milwaukee, WI, USA) with an M5S transducer according to the recommendations of the American Society of Echocardiography and the European Association of Echocardiography ${ }^{34}$. Pulsed-wave Doppler tissue imaging (DTI) of mitral and tricuspid annular motion was recorded at three annular sites in the apical fourchamber view; the values obtained at the septal and lateral sides of the mitral annulus were averaged. Diastolic function was graded as 0 (normal filling), 1 (impaired relaxation), 2 (pseudonormal filling) and 3 (restrictive filling). Echocardiographic examinations were recorded \pm 1 day 


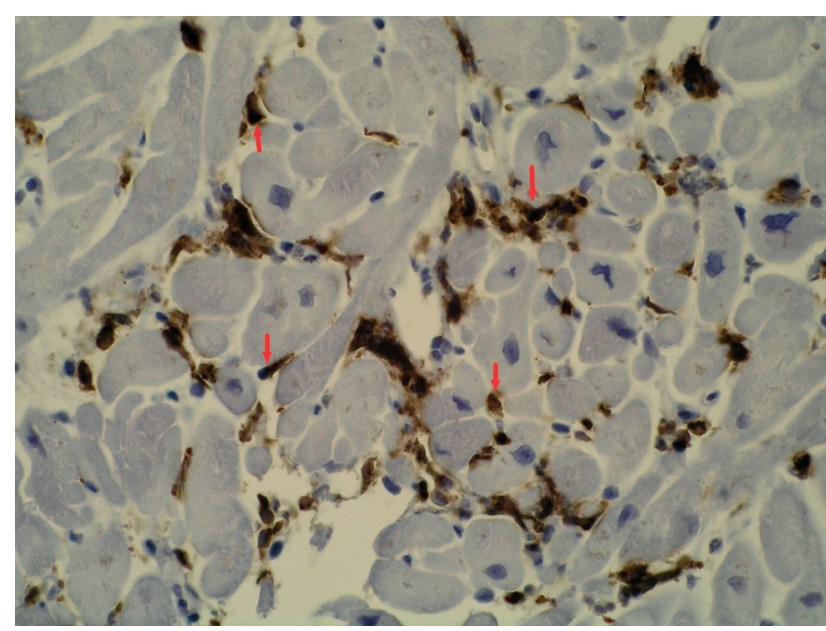

Fig. 1. Mononuclear leucocytes infiltration (LCA+ cells, imunohistochemistry, original magnification 200x).

from EMB and right heart catheterization (RHC). There was a follow up visit at 6 months \pm 14 days after baseline; clinical evaluation with an assessment of functional status was done at the same time. Functional status was graded according to NYHA classification ${ }^{35,36}$.

\section{Statistical analysis}

The values of all parameters at both visits and the change in these parameters were described as mean value, standard deviation, minimum and maximum. To assess the significance of the change in variables during the 6-month follow-up, a paired t-test or the nonparametric Wilcoxon signed-rank test was conducted (depending on data distribution). The difference between groups was compared using a t-test or Mann-Whitney test. Correlations were tested using Pearson's or Spearman's correlation coefficients (depending on data distribution). The association between baseline parameters and the improvement in LVEF was analyzed via univariate and multivariate logistic regression models. The parameters with potential predictive power (providing at least $P<0.1$ in univariate logistic regression) were included in the multivariate logistic regression models to identify independent predictors of improvement in LVEF. Results with a $P$-value $<0.05$ were considered statistically significant. The analyses were performed using SAS 9.3.

\section{RESULTS}

Improvement of LVEF function was found in 41 patients (i.e. $65 \%$ of whole group; I+ group). In this group, LVEF improved from $24 \pm 7 \%$ to $47 \pm 8 \%(P<0.001)$. In

Table 1. Comparison of the groups with and without LVEF increase.

\begin{tabular}{|c|c|c|c|c|c|c|c|c|}
\hline \multirow[b]{2}{*}{ Parameter } & & \multicolumn{3}{|c|}{$\begin{array}{l}\text { Group with LVEF increase (I+) } \\
\qquad(\mathrm{n}=41)\end{array}$} & \multicolumn{3}{|c|}{$\begin{array}{l}\text { Group without LVEF increase (I-) } \\
\qquad(\mathrm{n}=22)\end{array}$} & \multirow[b]{2}{*}{$P^{* *}$} \\
\hline & & Mean (SD) & Min-Max & $P^{*}$ & Mean (SD) & Min-Max & $P^{*}$ & \\
\hline \multicolumn{2}{|l|}{$\begin{array}{l}\text { Age } \\
\text { (years) }\end{array}$} & $44.3(11.73)$ & $19-67$ & & $43.1(12.68)$ & $22-69$ & & 0.699 \\
\hline \multicolumn{2}{|l|}{$\begin{array}{l}\text { Symptoms } \\
\text { (months) }\end{array}$} & $2.2(1.80)$ & $1-6$ & & $2.9(2.16)$ & $1-6$ & & 0.279 \\
\hline \multirow{3}{*}{$\begin{array}{l}\mathrm{LCA}+ \\
\left(\text { cells } / \mathrm{mm}^{2}\right)\end{array}$} & Baseline & $25.1(16.45)$ & $15-100$ & & $18.5(4.44)$ & $14-31$ & & 0.032 \\
\hline & After 6 months & $11.5(5.51)$ & $3-35$ & & $13.5(6.35)$ & $5-29$ & & 0.213 \\
\hline & Difference & $-13.6(14.30)$ & $-65-0$ & $<0.001$ & $-5.0(7.65)$ & $-19-15$ & 0.006 & 0.009 \\
\hline \multirow{3}{*}{$\begin{array}{l}\text { NTproBNP } \\
(\mathrm{ng} / \mathrm{L})\end{array}$} & Baseline & $3157.0(3126.07)$ & $90-11322$ & & $1525.8(1340.89)$ & $304-6465$ & & 0.065 \\
\hline & After 6 months & $345.9(417.55)$ & $32-1692$ & & $1488.1(2314.88)$ & $31-10912$ & & 0.001 \\
\hline & Difference & $-2871.3(3096.36)$ & $-11272-484$ & $<0.001$ & $-22.2(1349.95)$ & $-1639-4447$ & 0.240 & $<0.001$ \\
\hline \multirow[t]{3}{*}{ NYHA class } & Baseline & $2.52(0.602)$ & $1.0-3.5$ & & $2.36(0.560)$ & $1.5-3.5$ & & 0.242 \\
\hline & After 6 months & $1.43(0.396)$ & $1.0-2.0$ & & $1.95(0.596)$ & $1.0-3.0$ & & 0.001 \\
\hline & Difference & $-1.1(0.66)$ & $-2-0$ & $<0.001$ & $-0.4(0.53)$ & $-2-1$ & 0.003 & $<0.001$ \\
\hline \multirow{3}{*}{$\begin{array}{l}\mathrm{CI} \\
\left(\mathrm{L} / \mathrm{min} / \mathrm{m}^{2}\right)\end{array}$} & Baseline & $1.98(0.478)$ & $1.1-3.1$ & & $2.13(0.511)$ & $1.5-3.6$ & & 0.326 \\
\hline & After 6 months & $2.52(0.427)$ & $1.6-3.4$ & & $2.23(0.453)$ & $1.5-3.0$ & & 0.012 \\
\hline & Difference & $0.6(0.51)$ & $-1-2$ & $<0.001$ & $0.1(0.55)$ & $-1-1$ & 0.276 & 0.002 \\
\hline \multirow{3}{*}{$\begin{array}{l}\text { MPAP } \\
(\mathrm{mmHg})\end{array}$} & Baseline & $25.8(10.29)$ & $5-46$ & & $22.4(7.10)$ & $13-37$ & & 0.180 \\
\hline & After 6 months & $17.6(4.93)$ & $10-28$ & & $20.0(7.90)$ & $11-42$ & & 0.390 \\
\hline & Difference & $-8.3(9.62)$ & $-27-12$ & $<0.001$ & $-2.5(9.62)$ & $-19-19$ & 0.170 & 0.077 \\
\hline \multirow{3}{*}{$\begin{array}{l}\text { PCWP } \\
(\mathrm{mmHg})\end{array}$} & Baseline & $16.6(8.34)$ & $2-32$ & & $14.9(7.10)$ & $6-33$ & & 0.422 \\
\hline & After 6 months & $10.2(4.27)$ & $2-22$ & & $12.5(7.79)$ & $3-35$ & & 0.422 \\
\hline & Difference & $-6.5(8.64)$ & $-25-12$ & $<0.001$ & $-2.4(8.76)$ & $-21-17$ & 0.134 & 0.155 \\
\hline
\end{tabular}

*P - paired test (Wilcoxon rank sum text) for comparison of parameters at baseline and after 6 months

** $\mathrm{P}$ - Mann-Whitney test for comparison of parameters between groups

LVEF - left ventricular ejection fraction, LCA+ cells - leucocyte common antigen positive cells, CI - cardiac index, MPAP - mean pulmonary artery pressure, PCWP - pulmonary capillary wedge pressure 
Table 2. Comparison of echocardiographic parameters in the groups with and without LVEF increase.

\begin{tabular}{|c|c|c|c|c|c|c|c|c|}
\hline \multirow{2}{*}{\multicolumn{2}{|c|}{ Parameter }} & \multicolumn{3}{|c|}{$\begin{array}{l}\text { Group with LVEF increase }(\mathrm{I}+) \\
\qquad(\mathrm{n}=41)\end{array}$} & \multicolumn{4}{|c|}{$\begin{array}{l}\text { Group without LVEF increase (I-) } \\
\qquad(\mathrm{n}=22)\end{array}$} \\
\hline & & Mean (SD) & Min-Max & $P *$ & Mean (SD) & Min-Max & $P *$ & $P * *$ \\
\hline LVEF & Baseline & $23.7(7.29)$ & $15-40$ & & $26.1(7.19)$ & $17-38$ & & 0.171 \\
\hline \multirow[t]{2}{*}{$(\%)$} & After 6 months & $47.0(8.29)$ & $28-65$ & & $27.9(7.26)$ & $17-38$ & & $<0.001$ \\
\hline & Difference & $23.3(7.61)$ & $10-40$ & $<0.001$ & $1.8(5.56)$ & $-12-9$ & 0.128 & $<0.001$ \\
\hline LVESD & Baseline & $55.1(8.09)$ & $28-72$ & & $57.8(8.37)$ & $41-75$ & & 0.206 \\
\hline \multirow{2}{*}{$(\mathrm{mm})$} & After 6 months & $43.0(7.89)$ & $27-60$ & & $56.2(7.94)$ & $39-74$ & & $<0.001$ \\
\hline & Difference & $-12.1(6.41)$ & $-25-1$ & $<0.001$ & $-1.6(4.83)$ & $-12-6$ & 0.266 & $<0.001$ \\
\hline LVEDD & Baseline & $62.5(7.59)$ & $40-80$ & & $66.1(8.06)$ & $46-79$ & & 0.068 \\
\hline \multirow[t]{2}{*}{$(\mathrm{mm})$} & After 6 months & $55.5(7.45)$ & $40-72$ & & $64.9(7.12)$ & $54-79$ & & $<0.001$ \\
\hline & Difference & $-7.0(6.24)$ & $-23-7$ & $<0.001$ & $-1.2(5.09)$ & $-11-8$ & 0.412 & 0.001 \\
\hline RV & Baseline & $34.1(5.63)$ & $21-44$ & & $33.3(6.96)$ & $21-48$ & & 0.588 \\
\hline \multirow[t]{2}{*}{$(\mathrm{mm})$} & After 6 months & $31.9(4.61)$ & $24-44$ & & $32.5(5.47)$ & $18-40$ & & 0.430 \\
\hline & Difference & $-2.2(4.86)$ & $-14-9$ & 0.005 & $-0.8(6.64)$ & $-19-16$ & 0.383 & 0.461 \\
\hline LA & Baseline & $43.7(5.64)$ & $30-54$ & & $44.5(5.14)$ & $34-53$ & & 0.593 \\
\hline \multirow[t]{2}{*}{$(\mathrm{mm})$} & After 6 months & $39.8(6.21)$ & $26-55$ & & $42.5(5.64)$ & $28-56$ & & 0.058 \\
\hline & Difference & $-3.9(5.06)$ & $-12-9$ & $<0.001$ & $-2.0(4.72)$ & $-9-8$ & 0.065 & 0.159 \\
\hline s'mi & Baseline & $5.11(1.842)$ & $3.0-10.5$ & & $5.45(1.704)$ & $3.5-11.5$ & & 0.242 \\
\hline \multirow[t]{2}{*}{$(\mathrm{cm} / \mathrm{s})$} & After 6 months & $7.51(1.936)$ & $4.5-12.5$ & & $5.45(1.704)$ & $3.0-10.5$ & & $<0.001$ \\
\hline & Difference & $2.4(2.13)$ & $-2-7$ & $<0.001$ & $0.0(1.31)$ & $-3-2$ & 0.794 & $<0.001$ \\
\hline $\mathrm{e}^{\prime}$ & Baseline & $5.98(2.109)$ & $2.5-11.5$ & & 6.59 (1.919) & $4.0-10.0$ & & 0.230 \\
\hline \multirow[t]{2}{*}{$(\mathrm{cm} / \mathrm{s})$} & After 6 months & $7.74(2.213)$ & $2.5-14.0$ & & $5.86(1.891)$ & $2.5-11.0$ & & 0.001 \\
\hline & Difference & 1.7 (2.09) & $-3-6$ & $<0.001$ & $-0.7(2.41)$ & $-5-5$ & 0.167 & $<0.001$ \\
\hline \multirow[t]{3}{*}{ DF } & Baseline & $1.9(0.97)$ & $0-3$ & & $2.1(1.19)$ & $0-3$ & & 0.389 \\
\hline & After 6 months & $0.8(0.49)$ & $0-2$ & & $1.7(0.84)$ & $0-3$ & & $<0.001$ \\
\hline & Difference & $-1.1(1.06)$ & $-3-0$ & $<0.001$ & $-0.4(1.22)$ & $-2-3$ & 0.097 & 0.041 \\
\hline \multirow[t]{3}{*}{$\mathrm{E} / \mathrm{e}^{\prime}$} & Baseline & $14.08(7.887)$ & $4.6-42.7$ & & $13.81(4.888)$ & $6.0-28.8$ & & 0.395 \\
\hline & After 6 months & $8.21(2.737)$ & $3.8-17.8$ & & $12.44(3.895)$ & $5.4-21.2$ & & $<0.001$ \\
\hline & Difference & $-5.7(6.53)$ & $-25-6$ & $<0.001$ & $-1.4(5.66)$ & $-14-10$ & 0.318 & 0.027 \\
\hline \multirow{3}{*}{$\begin{array}{l}\mathrm{s}^{\prime} \text { tri } \\
(\mathrm{cm} / \mathrm{s})\end{array}$} & Baseline & $10.0(2.00)$ & $6-17$ & & $10.4(2.20)$ & $7-15$ & & 0.376 \\
\hline & After 6 months & $13.8(3.92)$ & $7-29$ & & $11.0(2.79)$ & $7-19$ & & 0.001 \\
\hline & Difference & $3.9(4.65)$ & $-6-20$ & $<0.001$ & $0.6(2.17)$ & $-3-4$ & 0.205 & 0.001 \\
\hline
\end{tabular}

$* P$ - paired test (Wilcoxon rank sum text) for comparison of parameters at baseline and after 6 months

** $P$ - Mann-Whitney test for comparison of parameters between groups

LVEF - left ventricular ejection fraction, LVESD - left ventricular end-systolic diameter, LVEDD - left ventricular end-diastolic diameter, RV - right ventricle, LA - left atrium DF - diastolic function, diastolic function was graded as 0 (normal filling), 1 (impaired relaxation), 2 (pseudonormal filling) and 3 (restrictive filling), E - early transmitral diastolic flow velocity (E wave), e' - diastolic mitral annular motion velocity assessed by pulsed-wave Doppler tissue imaging, s'mi - systolic mitral annular motion velocity assessed by pulsed-wave Doppler tissue imaging, s'tri - systolic tricuspid annular motion velocity assessed by pulsed-wave Doppler tissue imaging,

the remaining 22 patients (35\% of the group), there was no increase in LVEF of $\geq 10 \%$ (group I-); LVEF in this group changed from $26 \pm 7 \%$ to $28 \pm 8 \%(P=0.128)$. In the I+ group, a larger number of infiltrating LCA+ cells/ $\mathrm{mm}^{2}$ was detected in EMB sample at baseline $(25.1 \pm 16.5$ vs. $18.5 \pm 4.4$ cells $/ \mathrm{mm}^{2}$ in the I- group; $P=0.032$ ) and a decrease in the number of infiltrating LCA + in this group was more important (reduction of $13.6 \pm 14.3$ cells $/ \mathrm{mm}^{2}$ vs. $5.0 \pm 7.7$ cells $/ \mathrm{mm}^{2}$ in the I- group; $P=0.009$ ). More significant improvement in diastolic function assessed by a reduction in the $\mathrm{E} / \mathrm{e}^{\prime}$ ratio in $\mathrm{I}+$ group was observed (of $5.7 \pm 6.5$ in the I+ group vs. $1.4 \pm 5.7$ in the I- group; $P=0.027)$. Reduction in NYHA class was seen in both groups: in the I+ group from $2.5 \pm 0.6$ to $1.4 \pm 0.4(P<$ $0.001)$ and from $2.4 \pm 0.6$ to $2.0 \pm 0.6(P=0.003)$ in the I- group. The difference between the groups was highly statistically significant $(P<0.001)$. Also a decrease in the levels of NTproBNP and an improvement in other echocardiographic and hemodynamic parameters obtained by RHC was more pronounced in group I+ (see Table 1 and 2).

In the univariate logistic regression at the $10 \%$ level of significance, the following predictors of improved LVEF were identified: number of LCA+ cells $(\mathrm{P}=0.089)$, end-diastolic diameter of LV $(\mathrm{P}=0.088)$ and NTproBNP value $(\mathrm{P}=0.046)$ at the time of diagnosis. In the multivariate analysis, only NTproBNP value was confirmed as an independent predictor of LVEF improvement $(\mathrm{OR}=1.2$; 1.003 to $1.394 ; \mathrm{P}=0.046$ ).

The change in NTproBNP value correlated significantly with the change in hemodynamic parameters particularly strong in the I- group; strong correlation with mean pulmonary artery pressure (MPAP) was found (r $=0.77, P<0.001)$. Similarly, correlation with change in 
Table 3. Corelations parameters changes in $\mathrm{I}+\operatorname{group}(\mathrm{n}=41)$.

\begin{tabular}{|c|c|c|c|c|c|c|c|c|c|c|c|c|c|c|}
\hline & \multicolumn{2}{|c|}{$\Delta$ NYHA } & \multicolumn{2}{|c|}{$\Delta$ LVEF } & \multicolumn{2}{|c|}{$\Delta$ NTpr oBNP } & \multicolumn{2}{|c|}{$\Delta \mathrm{LCA}+$ cells } & \multicolumn{2}{|c|}{$\Delta \mathrm{PCWP}$} & \multicolumn{2}{|c|}{$\Delta \mathrm{CI}$} & \multicolumn{2}{|c|}{$\Delta$ MPAP } \\
\hline & $\mathrm{r}$ & $P$ & $\mathrm{r}$ & $P$ & $\mathrm{r}$ & $P$ & $\mathrm{r}$ & $P$ & $\mathrm{r}$ & $P$ & $\mathrm{r}$ & $P$ & $\mathrm{r}$ & $P$ \\
\hline$\Delta \mathrm{CI}$ & -0.26 & & $r$ & & -0.46 & 0.004 & -0.32 & 0.044 & -0.49 & 0.001 & & & -0.45 & 0.00 \\
\hline$\Delta \mathrm{L}$ & 33 & & 10 & & & .342 & -0.20 & 0.202 & 0.20 & & -0.04 & & 0.11 & 0.505 \\
\hline$\Delta \mathrm{DF}$ & 0.39 & 0.012 & .13 & 0.4 & 0.34 & 0.037 & 0.23 & 0.152 & 0.65 & $<0.001$ & -0.49 & & 0.63 & $<0.001$ \\
\hline & .34 & 0.030 & - & - & -0.28 & 0.080 & -0.24 & 0.127 & -0.26 & & 0.17 & & -0.16 & 0.317 \\
\hline & & & 0.41 & & & & -0.04 & & 8 & & & & 6 & \\
\hline$\Delta \mathrm{E} / \mathrm{e}$ & 0.08 & 0.612 & -0.13 & 0.432 & 0.21 & 0.215 & 0.21 & 0.183 & 0.55 & $<0.001$ & -0.38 & & 0.56 & $<0.001$ \\
\hline$\Delta \mathrm{LCA}$ & .15 & 0.336 & -0.24 & & & 0.347 & - & - & 0.26 & 0.110 & -0.32 & & 0.27 & 0.089 \\
\hline$\Delta \mathrm{LA}$ & 0.35 & 0.025 & -0.26 & 0. & 0.30 & 0.060 & -0.14 & 0.395 & 0.39 & & -0.10 & & 0.34 & 0.032 \\
\hline$\Delta \mathrm{N}$ & .38 & 0.016 & -0.16 & 0.317 & 0.31 & 0.056 & 0.27 & 0.089 & 0.92 & $<0.001$ & -0.45 & & - & - \\
\hline$\Delta \mathrm{N}$ & 0.60 & $<0.001$ & -0.28 & & - & - & 0.15 & 0.347 & 0.39 & & -0.46 & & 0.31 & 0.05 \\
\hline$\Delta \mathrm{N}$ & - & - & 0.34 & 0.030 & 0.60 & $<0.001$ & 0.15 & 0.336 & 0.43 & 0.006 & -0.26 & & 0.38 & 0.016 \\
\hline & 0.43 & & -0.26 & & & 0.014 & 0.26 & & - & - & & & 0.92 & $<0.001$ \\
\hline$\Delta \mathrm{RV}$ & 0.18 & 0.250 & -0.20 & 0.213 & 0.46 & 0.003 & 0.14 & 0.398 & 0.32 & 0.041 & -0.47 & 0.002 & 0.26 & 0.10 \\
\hline$\Delta \mathrm{s}^{\prime} \mathrm{mi}$ & -0.24 & 0.138 & 0.39 & 0.012 & -0.21 & 0.205 & -0.14 & 0.383 & -0.54 & $<0.001$ & 0.41 & 0.011 & -0.55 & $<0.001$ \\
\hline$\Delta$ s'tri & -0.38 & 0.014 & 0.38 & 0.016 & -0.48 & 0.002 & -0.24 & 0.127 & -0.46 & 0.003 & 0.46 & 0.003 & -0.46 & 0.003 \\
\hline
\end{tabular}

$\mathrm{r}=$ correlation coefficient

$P=\mathrm{p}$-value of correlation coefficient

CI - cardiac index, DF - diastolic function, diastolic function was graded as 0 (normal filling), 1 (impaired relaxation), 2 (pseudonormal filling) and 3 (restrictive filling), LVEF - left ventricular ejection fraction, LVEDD - left ventricular end-diastolic diameter, LVESD - left ventricular end-systolic diameter, LA - left atrium, E - early transmitral diastolic flow velocity (E wave), e' - diastolic mitral annular motion velocity assessed by pulsed-wave Doppler tissue imaging, s'mi - systolic mitral annular motion velocity assessed by pulsed-wave Doppler tissue imaging, s'tri - systolic tricuspid annular motion velocity assessed by pulsed-wave Doppler tissue imaging, RV - right ventricle, MPAP - mean pulmonary artery pressure, PCWP - pulmonary capillary wedge pressure

Table 4. Corelations parameters changes in I- group $(n=22)$.

\begin{tabular}{|c|c|c|c|c|c|c|c|c|c|c|c|c|c|c|}
\hline & \multicolumn{2}{|c|}{$\Delta$ NYHA } & \multicolumn{2}{|c|}{$\Delta$ LVEF } & \multicolumn{2}{|c|}{$\Delta$ NTproBNP } & \multicolumn{2}{|c|}{$\Delta \mathrm{LCA}+$ cells } & \multicolumn{2}{|c|}{$\Delta \mathrm{PCWP}$} & \multicolumn{2}{|c|}{$\Delta \mathrm{CI}$} & \multicolumn{2}{|c|}{$\Delta$ MPAP } \\
\hline & $\mathrm{r}$ & $P$ & $\mathrm{r}$ & $P$ & $\mathrm{r}$ & $P$ & $\mathrm{r}$ & $P$ & $\mathrm{r}$ & $P$ & $\mathrm{r}$ & $P$ & $\mathrm{r}$ & $P$ \\
\hline$\Delta \mathrm{CI}$ & -0.30 & 0.168 & 0.51 & 0.016 & -0.64 & 0.002 & -0.24 & 0.289 & -0.47 & 0.026 & - & - & -0.50 & 0.019 \\
\hline$\Delta$ LVEDD & -0.18 & 0.422 & -0.15 & 0.511 & 0.14 & 0.545 & -0.02 & 0.913 & 0.21 & 0.353 & -0.30 & 0.173 & 0.24 & 0.275 \\
\hline$\Delta \mathrm{DF}$ & -0.05 & 0.823 & -0.39 & 0.071 & 0.25 & 0.269 & -0.10 & 0.661 & 0.43 & 0.046 & -0.17 & 0.449 & 0.49 & 0.022 \\
\hline$\Delta$ LVEF & -0.16 & 0.468 & - & - & -0.47 & 0.031 & -0.16 & 0.489 & -0.55 & 0.009 & 0.51 & 0.016 & -0.44 & 0.038 \\
\hline$\Delta$ LVESD & -0.10 & 0.659 & -0.51 & 0.016 & 0.32 & 0.160 & 0.01 & 0.948 & 0.48 & 0.022 & -0.33 & 0.133 & 0.45 & 0.037 \\
\hline$\Delta \mathrm{E} / \mathrm{e}$ & -0.06 & 0.780 & -0.18 & 0.418 & 0.35 & 0.125 & 0.27 & 0.232 & 0.43 & 0.045 & -0.23 & 0.306 & 0.48 & 0.024 \\
\hline$\Delta \mathrm{LCA}+$ cells & -0.09 & 0.698 & -0.16 & 0.489 & 0.02 & 0.924 & - & - & 0.13 & 0.563 & -0.24 & 0.289 & 0.08 & 0.726 \\
\hline$\Delta \mathrm{LA}$ & 0.12 & 0.581 & -0.64 & 0.001 & 0.64 & 0.002 & -0.01 & 0.968 & 0.57 & 0.006 & -0.54 & 0.010 & 0.58 & 0.005 \\
\hline$\Delta$ MPAP & 0.09 & 0.680 & -0.44 & 0.038 & 0.77 & $<0.001$ & 0.08 & 0.726 & 0.95 & $<0.001$ & -0.50 & 0.019 & - & - \\
\hline$\Delta$ NTproBNP & 0.39 & 0.084 & -0.47 & 0.031 & - & - & 0.02 & 0.924 & 0.72 & $<0.001$ & -0.64 & 0.002 & 0.77 & $<0.001$ \\
\hline$\Delta$ NYHA & - & - & -0.16 & 0.468 & 0.39 & 0.084 & -0.09 & 0.698 & 0.08 & 0.724 & -0.30 & 0.168 & 0.09 & 0.680 \\
\hline$\Delta \mathrm{PCWP}$ & 0.08 & 0.724 & -0.55 & 0.009 & 0.72 & $<0.001$ & 0.13 & 0.563 & - & - & -0.47 & 0.026 & 0.95 & $<0.001$ \\
\hline$\Delta \mathrm{RV}$ & 0.02 & 0.922 & 0.00 & 0.987 & 0.52 & 0.016 & 0.20 & 0.382 & 0.42 & 0.050 & -0.35 & 0.111 & 0.43 & 0.047 \\
\hline$\Delta$ s'mi $^{\prime}$ & - & - & 0.57 & 0.006 & -0.46 & 0.034 & -0.41 & 0.056 & -0.77 & $<0.001$ & 0.48 & 0.025 & -0.74 & $<0.001$ \\
\hline$\Delta$ s'tri $^{\prime}$ & 0.08 & 0.738 & -0.21 & 0.351 & 0.23 & 0.312 & -0.55 & 0.009 & 0.19 & 0.399 & 0.13 & 0.560 & 0.14 & 0.525 \\
\hline
\end{tabular}

$\mathrm{r}_{=}$correlation coefficient

$P=$ p-value of correlation coefficient

CI - cardiac index, DF - diastolic function, diastolic function was graded as 0 (normal filling), 1 (impaired relaxation), 2 (pseudonormal filling) and 3 (restrictive filling), LVEF - left ventricular ejection fraction, LVEDD - left ventricular end-diastolic diameter, LVESD - left ventricular end-systolic diameter, LA - left atrium, E - early transmitral diastolic flow velocity (E wave), e' - diastolic mitral annular motion velocity assessed by pulsed-wave Doppler tissue imaging, s'mi - systolic mitral annular motion velocity assessed by pulsed-wave Doppler tissue imaging, s'tri - systolic tricuspid annular motion velocity assessed by pulsed-wave Doppler tissue imaging,RV - right ventricle, MPAP - mean pulmonary artery pressure, PCWP - pulmonary capillary wedge pressure 
pulmonary capillary wedge pressure (PCWP; $\mathrm{r}=0.72, P$ $<0.001)$ and change of cardiac index $(\mathrm{CI} ; \mathrm{r}=-0.64, P=$ 0.002 ) was found. In the group I+, correlations of the parameter change with changes in NTproBNP change were less tight or even nonsignificant (for MPAP: $\mathrm{r}=0.31, P$ $=0.056$; for PCWP: $\mathrm{r}=0.39, P=0.014$; and for CI: $\mathrm{r}=$ $-0.46, P=0.004)$. Improvement of the echocardiographic parameters of diastolic function in both groups correlated with a decrease in the PCWP and MPAP. Improved RV systolic function (assessed by the velocity of tricuspid annular systolic motion) was statistically significant only in the I+ group and correlated with a decrease in the MPAP and PCWP, NTproBNP and NYHA class. The correlations are shown in Tables 3 and 4.

\section{DISCUSSION}

LV systolic function improvement is relatively often observed in patients with RODCM (ref. ${ }^{28,32,37}$ ). This improvement is more pronounced in patients with proven myocarditis $^{25}$. Hence, it is possible that the majority of improvements in LV function in the whole group of RODCM without evaluation of myocardial inflammation is based, in the main, on improvement in patients with myocarditis (ICM). The reversibility of myocardial dysfunction in a majority of ICM cases confirms that such disorder is not caused just by irreversible loss of myocytes or permanent loss of integrity of the extracellular matrix. Defective myocardial contractility is most probably also caused by production of negative inotropic mediators associated with inflammation, such as cytokines (i.e. tumor necrosis factor alpha, interferons, interleukins) (ref. ${ }^{2}$ ).

We also observed a small improvement in LVEF in the group without biopsy-proven inflammation in our previous study, and speculate that several cases where a significant improvement in LVEF is observed despite an $\mathrm{LCA}+$ cell count of $\leq 14 / \mathrm{mm}^{2}$, may be caused by falsenegative biopsy findings. It is known that EMB is highly specific but far from being $100 \%$ sensitive due to possible focal distribution of inflammatory changes. An interesting study by Chow et al. reported that by using histological evaluation of EMB, which is less sensitive than immunohistochemical methods, taking 8-9 samples achieved "only" $79 \%$ sensitivity ${ }^{38}$.

The published data as well as clinical experience show that even the group with biopsy proven ICM is not homogenous and even in this setting there is a group of patients with no improvement in LVEF. In our study, we compared the differences between ICM subgroups with and without the improvement in LV systolic function.

First and foremost, we confirmed LV systolic function improvement in 2/3 of patients with ICM which corresponds well with published data ${ }^{4,5,7-10,28}$. In the subgroup with improved LVEF (I+ group), which we defined as at least a $10 \%$ increase in LVEF, an actual LVEF increase by $23 \pm 8 \%$ on average was seen. Not surprisingly, the LVEF value in the I- group (patients with LVEF recovery $<10 \%$ or without any improvement) remained virtually unchanged. As might be expected, increase in LVEF was followed by improvement in a number of other echocardiographic parameters, including indicators of diastolic LV function and systolic function of RV. Likewise, significant decrease in NTproBNP and the NYHA class and significantly better evolution of hemodynamic parameters in the I+ group were described. In group I-, some decrease in MPA and PCW occurred as well, however, the difference in favor of group I+ for these parameters was not statistically significant in contrast to the increase of CI.

A significant difference between groups was also found for the number of infiltrating LCA+ cells at diagnosis which we regard as a correlate of inflammation severity. In group $\mathrm{I}+$, on average $25.1 \pm 16.5$ cells $/ \mathrm{mm}^{2}$ were detected, while in group I- only $18.5 \pm 4.4$ cells $/ \mathrm{mm}^{2}(P=$ 0.032 ) were found. One recent paper reported that an increasing number of LCA+ cells has a negative prognostic significance ${ }^{29}$. On the other hand, it is known that during fulminant myocarditis where the number of infiltrating cells may be over $100 / \mathrm{mm}^{2}$, the marked inflammatory response can be replaced by rapid retreat of inflammatory infiltration associated with significant improvement in LV function. In our study, a decrease in the number of infiltrating LCA+ cells which we consider a sign of inflammation healing, was observed in both groups: in the I+ group with LVEF increase, we found a decline of $13.6 \pm 14.3$ cells $/ \mathrm{mm}^{2}(P<0.001)$ and in the I- group with no improved LVEF, there was a decline in the number of LCA + cells of $5.0 \pm 7.7$ cells $/ \mathrm{mm}^{2}(P=0.006)$. This created a significant difference in favor of the I+ group $(P=0.009)$. We therefore presumed that a larger baseline number of LCA+ cells could be a predictor of LVEF improvement. This was confirmed in the univariate analysis at the $10 \%$ level of significance. Here, apart from the number of LCA+ cells $(P=0.089)$, two other predictors were identified: $\mathrm{LV}$ diastolic dimension $(P=0.088)$ and particularly the NTproBNP value $(P=0.046)$ LV dilatation is generally regarded as a negative predictor of LV function recovery ${ }^{39,40}$, which corresponds to our findings. Conversely, a higher NTproBNP level is considered a negative prognostic indicator ${ }^{41}$, which was not confirmed by our observations. Using multivariate regression, the only significant predictor of LVEF change was baseline NTproBNP. A higher baseline value of NTproBNP by 500 $\mathrm{ng} / \mathrm{L}$ means a $20 \%$ higher chance of increase in LVEF of at least $10 \%$ after a 6 -month follow-up.

From the results, NTproBNP emerged as a significant indicator of further development as well, but in the opposite direction than might be expected. A possible explanation of this surprising finding may be that patients in group I+ had a 2-fold higher baseline NTproBNP value $(3157 \pm 3126 \mathrm{ng} / \mathrm{L}$ vs. $1526 \pm 1341 \mathrm{ng} / \mathrm{L}$ in the I- group; $P=0.065)$. However, LVEF was similar in both groups, which could indicate that the heart failure therapy was not optimally set. Reserve in the dosing of pharmacological treatment in the I+ group may be a reason increasing the chance of recovery. This hypothesis is supported by the RHC data, where higher baseline values of PCWP and MPAP, and also lower CI, were found in the I+ group, even though the differences between groups were not statistically significant. However, this may be associated 
with small sample size, which is one of the limitations of our study. Another fact that supports this theory is that all 3 patients with NTproBNP values of over 10000 ng/L were part of the group I+. All of these patients were also in a clinically very serious condition at hospital admission with the need for intravenous therapy. If these three patients were excluded from analysis, the average NTproBNP value in I+ group would be reduced to 2531 $\pm 2277 \mathrm{ng} / \mathrm{L}$, which is still higher than in I- group (1526 \pm $1341 \mathrm{ng} / \mathrm{L})$, but not significantly so $(P=0.145)$. It is also notable that the LVEF and NYHA class improvements in these three individuals were higher than the average of the I+ group.

MPAP and PCWP decline and also CI rise were significant only in group I+ at follow-up control. Comparing changes in hemodynamic parameters between the groups, only the difference of CI reached statistical significance. Changes in hemodynamic parameters also correlated with changes in the the echocardiographic parameters of diastolic function in both groups, but more closely in I+. In this group, correlation of the change in grade of diastolic function and PCWP change was significant ( $\mathrm{r}=0.65, P<$ 0.001 ); correlation of PCWP change with the change of the ratio $\mathrm{E} / \mathrm{e}^{\prime}$ was also statistically significant $(\mathrm{r}=0.55, P$ $<0.001)$. Decrease in functional NYHA class correlated with a decrease of NTproBNP and PCWP in the I+ group (unlike I-).

This leads us to a hypothesis explaining the fact that higher NTproBNP level was found a positive predictor of improvement of LV function. Optimizing of heart failure therapy dosing after initial examination can probably be one of the factors causing more significant improvement in some patients with ICM. Despite the fact that at least a 14-day heart failure therapy was required in all patients prior to performing baseline EMB and RHC, this may not be sufficient time to set the optimum dosage. Symptoms duration in the I+ group was also shorter than in I- group ( $2.2 \pm 1.8$ vs $2.9 \pm 2.2$ months), but without statistical significance. It can therefore be assumed that the optimal pharmacological treatment of heart failure according to guidelines can cause a positive effect in patients with ICM although this has not been convincingly demonstrated in a placebo-controlled study yet. In agreement with this, it was suggested that the increasing chance for LV function improvement in recent studies compared with older data was associated with advances in pharmacological therapy ${ }^{42}$. Also some experimental data as well as clinical studies confirm this. The positive effect of administration of the ACE-inhibitors/angiotensin receptor blockers (ARBs) has been demonstrated in an animal model of myocarditis ${ }^{43,44}$; the same was true also for aldosterone receptor antagonists ${ }^{45}$. Similarly, a better prognosis was described after beta-blocker therapy in the animal model and also in a human study ${ }^{31,46}$.

The proportion of patients on basic heart failure drugs was similar in both groups: in the I+ group ACE-inhibitors / ARBs in $96 \%$ of the patients and in the I- group in $98 \%$; beta-blockers in $98 \%$ and $96 \%$; MRA in $82 \%$ and $85 \%$, furosemide in both groups in $87 \%$.
Another exciting topic is immunosuppressive therapy in ICM. According to some studies and meta-analyses the administration of immunosuppression may be associated with a greater chance of LV function improvement ${ }^{15,16}$, especially in patients with symptoms of more than 6 months ${ }^{47}$, without viral presence in the myocardium and with positive autoantibodies ${ }^{48}$. In our study, the average time from onset of symptoms to diagnostic EMB was approximately 2.5 months. So this was a group without any evidence for such therapeutic approach, since it was not used in these patients.

We see the importance of early EMB-based differential diagnostics of RODCM, inter alia, in the fact, that in the group with inflammation (ICM patients) a significant improvement in LVEF was found in a 3-6 month interval, which was not the case in the non-inflammatory DCM. So altogether, the result of the biopsy is crucial for further therapeutic decisions and their timing (i.e. for ICD implantation and/or cardiac resynchronization therapy introduction, inclusion on the waiting list for heart transplantation). Finally, the threshold for significant improvement of LVEF in our study was chosen with respect to the baseline value of LVEF (around 25\%), where the improvement exceeding $10 \%$ precludes indications for ICD implantation in primary prevention of sudden cardiac death $^{35,36}$. As described previously, it is no uniform course for every ICM case. For this reason it would be obviously useful to be able to accurately distinguish the groups of ICM patients where LVEF increase can be expected and where the improvement is less probable. The results of this study could help in the stratification of clinical course of ICM patients with several therapeutic consequences.

\section{CONCLUSION}

We found an improvement in LV systolic function in around two thirds of ICM patients associated with a significant improvement in a number of other parameters compared with patients who showed no LVEF increase. A higher NTproBNP value was the only independent predictor of LVEF amelioration. A change in NTproBNP levels was associated with changes in the majority of hemodynamic, echocardiographic and clinical parameters. Although there was a significantly larger number of inflammatory cells in the myocardium of the group with LV function improvement and the number of LCA+ cells at baseline showed a trend for predicting LVEF improvement, this was not confirmed in a multivariate regression. Similarly, a decrease in the number of LCA + cells was more pronounced in I+ group but this not correlated significantly with changes in most of the parameters.

Acknowledgement: This study was supported by grant IGA MZ CR 14087-3/2013 and by Specific University Research Grant MUNI/A/1270/2015.

Author contributions: JK: study concept and design; acquisition of data, data analysis and interpretation, drafting of the manuscript; PH, EO, DM, LS, VZ, IS: acquisition of data, data analysis and interpretation, critical revision of 
the manuscript; RS: study concept and design, analysis and statistical evaluation of data, critical revision of the manuscript.

Conflicts of interest statement: The authors declare there are no conflicts of interest regarding the publication of this article.

\section{REFERENCES}

1. Stehlik J, Edwards LB, Kucheryavaya AY, Aurora P, Christie JD, Kirk R, Dobbels F, Rahmel AO, Hertz MI. The Registry of the International Society for Heart and Lung Transplantation: twenty-seventh official adult heart transplant report-2010. J Heart Lung Transplant 2010;29:1089-103

2. Kühl U. Antiviral treatment of myocarditis and acute dilated cardiomyopathy. Heart Failure Clin 2005;1:467-74.

3. Hufnagel G, Pankuweit S, Richter A, Schonian U, Maisch B. The European Study of Epidemiology and Treatment of Cardiac Inflammatory Diseases (ESETCID): first epidemiological results. Herz 2000;25:279-85.

4. Caforio ALP, Pankuweit S, Arbustini E, Basso C, Gimeno-Blanes J, Felix SB, Fu M, Heliö T, Heymans S, Jahns R, Klingel K, Linhart A, Maisch B, McKenna W, Mogensen J, Pinto YM, Ristic A, Schultheiss HP, Seggewiss H, Tavazzi L, Thiene G, Yilmaz A, Charron P, Elliott PM. Current state of knowledge on aetiology, diagnosis, management, and therapy of myocarditis: a position statement of the European Society of Cardiology Working Group on Myocardial and Pericardial Diseases. Eur Heart J 2013;34:2636-48.

5. Cooper LT. Myocarditis. N Engl J Med 2009;360:1526-38.

6. Caforio AL, Calabrese F, Angelini A, Tona F, Vinci A, Bottaro S, Ramondo A, Carturan E, Iliceto S, Thiene G, Daliento L. A prospective study of biopsy-proven myocarditis: prognostic relevance of clinical and aetiopathogenetic features at diagnosis. Eur Heart J 2007;28:1326-33.

7. Kindermann I, Barth C, Mahfoud F, Ukena C, Lenski M, Yilmaz A, Klingel K, Kandolf R, Sechtem U, Cooper LT, Böhm M. Update on myocarditis. J Am Coll Cardiol 2012;59:779-92.

8. Dennert R, Crijns HJ, Heymans S. Acute viral myocarditis. Eur Heart J 2008;29:2073-82.

9. Schultheiss HP, Kühl U, Cooper LT. The management of myocarditis. Eur Heart J 2011;32:2616-25.

10. Caforio ALP, Marcolongo R, Basso C, Iliceto S. Clinical presentation and diagnosis of myocarditis. Heart 2015;101:1332-44.

11. Richardson P, McKenna W, Bristow M, Maisch B, Mautner B, O'Connell J, Olsen E, Thiene G, Goodwin J, Gyarfas I, Martin I, Nordet P. Report of the 1995 World Health Organisation/International Society and Federation of Cardiology Task Force on the Definition and Classification of cardiomyopathies. Circulation 1996;93:841-42.

12. Aretz HAT, Billingham ME, Edwards WD, Factor SM, Fallon JT, Fenoglio JJ Jr, Olsen EG, Schoen FJ. Myocarditis. A histopathologic definition and classification. Am J Cardiovasc Pathol 1987;1:3-14.

13. Kühl U, Noutsias M, Seeberg B, Schultheiss HP. Immunohistological evidence for chronic intramyocardial inflammatory proces in dilated cardiomyopathy. Heart 1996;75:295-300.

14. Maisch B, Portig I, Ristic A, Hufnagel G, Pankuweit S. Definition of inflammatory cardiomyopathy (myocarditis): on the way to consensus. A status report. Herz 2000;25:200-9.

15. Wojnicz R, Nowalany-Kozielska E, Wojciechowska C, Glanowska G, Wilczewski P, Niklewski T, Zembala M, Polonski L, Rozek MM Wodniecki J. et al. Randomized, placebo controled study for immunosuppressive treatment of inflammatory dilated cardiomyopathy. Circulation 2001;104:39-45.

16. Frustaci $A$, Russo MA, Chimenti $C$. Randomized study on the efficacy of immunosuppressive therapy in patients with virus-negative inflammatory cardiomyopathy: the TIMIC study. Eur Heart J 2009;30:1995-2002.

17. Thiene G, Bruneval P, Veinot J, Leone O. Diagnostic use of endomyocardial biopsy: a consensus statement. Virchows Arch 2013;463:1-5.

18. Palecek T, Kuchynka P, Hulinska D, Schramlova J, Hrbackova $H_{\text {, }}$ Vitkova I, Simek S, Horak J, Louch WE, Linhart A. Presence of Borrelia burgdorferi in endomyocardial biopsies in patients with new-on- set unexplained dilated cardiomyopathy. Med Microbiol Immunol 2010;199:139-43.

19. Kubanek $M$, Sramko $M$, Berenova $D$, Hulínská $D$, Hrbáčková $H$ Malušková J, Lodererová A, Málek I, Kautzner J. Detection of Borrelia burgdorferi sensu lato in endomyocardial biopsy specimens in individuals with recent-onset dilated cardiomyopathy. Eur J Heart Fail 2012;14:588-96.

20. Kuchynka P, Palecek T, Grus T, Lindner J, Berenova D, Kurzova Z, Balatova P, Krsek D, Vitkova I, Nemecek E, Podzimkova J, Danek AB, Linhart A. Absence of Borrelia burgdorferi in the myocardium of subjects with normal left ventricular systolic function: a study using PCR and electron microscopy. Biomed Pap Med Fac Univ Palacky Olomouc Czech Repub 2016;160:136-9.

21. Caforio ALP, Bottaro S, Iliceto S. Dilated cardiomyopathy and myocarditis: Classification, clinical and autoimmune features. Applied Cardiopulmonary Pathophysiology 2012;16:82-95.

22. Wehlou C, Delanghe JR. Detection of antibodies in cardiac autoimmmunity. Clinica Chimica Acta 2009;408:114-22.

23. Krejci J, Poloczkova H, Hude P, Freiberger T, Nemcova E, Zampachova V, Sirotkova A, Stepanova R, Spinarova L, Nemec P, Vitovec J. Impact of inflammatory infiltration and viral genome presence in myocardium on the changes of echocardiographic parameters. Cor et Vasa 2013;55:e333-e340.

24. Kühl U, Pauschinger M, Noutsias M, Seeberg B, Bock T, Lassner D, Poller W, Kandolf R, Schultheiss HP. High prevalence of viral genomes and multiple viral infections in the myocardium of adults with „idiopathic" left ventricular dysfunction. Circulation 2005;111:887-93.

25. Krejci J, Hude P, Poloczkova H, Zampachova V, Stepanova R, Freiberger T, Nemcova E, Spinarova L. Correlations of the changes in bioptic findings with echocardiographic, clinical and laboratory parameters in patients with inflammatory cardiomyopathy. Heart Vessels 2016;31:416-26.

26. McCarthy RE, Boehmer JP, Hruban RH, Hutchins GM, Kasper EK, Hare JM, Baughman KL. Long-term outcome of fulminant myocarditis as compared with acute (nonfulminant) myocarditis. N Engl J Med 2000;342:690-95

27. Corsten MF, Schroen B, Heymans S. Inflammation in viral myocarditis: friend or foe? Trends Molecular Med 2012;18:426-37.

28. D’Ambrosio A, Patti G, Manzoli A, Sinagra G, Di Lenarda A, Silvestri F, Di Sciascio $G$. The fate of acute myocarditis between spontaneous improvement and evolution to dilated cardiomyopathy: a review. Heart 2001;85:499-504.

29. Escher F, Kühl U, Lassner D, Stroux A, Westermann D, Skurk C, Tschöpe C, Poller W, Schultheiss HP. Presence of perforin in endomyocardial biopsies of patients with inflammatory cardiomyopathy predicts poor outcome. Eur J Heart Fail 2014;16:1066-72.

30. Fairweather D, Cooper LT, Blauwet LA. Sex and gender differences in myocarditis and dilated cardiomyopathy. Curr Probl Cardiol 2013;38:7-46.

31. Kindermann I, Kindermann M, Kandolf R, Klingel K, Bültmann B, Müller T, Lindinger A, Böhm M. Predictors of outcome in patients with suspected myocarditis. Circulation 2008;118:639-48.

32. Cooper LT, Mather PJ, Alexis JD, Pauly DF, Torre-Amione G, Wittstein IS, Dec GW, Zucker M, Narula J, Kip K, McNamara DM. Myocardial recovery in peripartum cardiomyopathy: prospective comparison with recent onset cardiomyopathy in men and nonperipartum women. J Cardiac Fail 2012;18:28-33.

33. Maisch B, Pankuweit S. Current treatment options in (peri)myocarditis and inflammatory. Herz 2012;37:644-56.

34. Lang RM, Badano LP, Mor-Avi V, Afilalo J, Armstrong A, Ernande L, Flachskampf FA, Foster E, Goldstein SA, Kuznetsova T, Lancellotti P, Muraru D, Picard MH, Rietzschel ER, Rudski L, Spencer KT, Tsang W, Voigt JU. Recommendation for cardiac chambre quantificantions by echocardiography in adults: An update from the American Society of Echocardiography and the European Association of Cardiocascular Imaging. Eur Heart J Cardiovasc Imaging 2015;16:233-70.

35. McMurray JJ, Adamopoulos S, Anker SD, Auricchio A, Böhm M, Dickstein K, Falk V, Filippatos G, Fonseca C, Gomez-Sanchez MA, Jaarsma T, Køber L, LipGY, Maggioni AP, Parkhomenko A, Pieske BM, Popescu BA, Rønnevik PK, Rutten FH, Schwitter J, Seferovic P, Stepinska J, Trindade PT, Voors AA, Zannad F, Zeiher A. ESC Guidelines for the diagnosis and treatment of acute and chronic heart failure 2012: The Task Force for the Diagnosis and Treatment of Acute and Chronic Heart Failure 2012 of the European Society 
of Cardiology. Developed in collaboration with the Heart Failure Association (HFA) of the ESC. Eur Heart J 2012;33:1787-847.

36. Yancy CW, Jessup M, Bozkurt B, Butler J, Casey DE, Drazner MH, Fonarow GC, Geraci SA, Horwich T, Januzzi JL, Johnson MR, Kasper EK, Levy WC, Masoudi FA, McBride PE, McMurray JJ, Mitchell JE, Peterson PN, Riegel B, Sam F, Stevenson LW, Tang WH, Tsai EJ, Wilkoff BL. 2013 ACCF/AHA guideline for the management of heart failure: a report of the American College of Cardiology Foundation/ American Heart Association Task Force on Practice Guidelines. J Am Coll Cardiol 2013;62:e147-239.

37. Kubanek M, Sramko M, Maluskova J, Kautznerova D, Weichet J, Lupinek P, Vrbska J, Malek I, Kautzner J. Novel predictors of left ventricular reverse remodelling in individuals with recent onset dilated cardiomyopathy. J Am Coll Cardiol 2013;61:54-63.

38. Chow LH, Radio SJ, Sears TD, McManus BM. Insensitivity of right ventricular endomyocardial biopsy in the diagnosis of myocarditis. J Am Coll Cardiol 1989;14:915-20.

39. Felker GM, Boehmer JP, Hruban RH, Hutchins GM, Kasper EK, Baughman KL, Hare JM. Echocardiographic findings in fulminant and acute myocarditis. J Am Coll Cardiol 2000;36:227-32.

40. Magnani JW, Dec GW. Myocarditis Current Trends in Diagnosis and Treatment. Circulation. 2006 Feb 14;113:876-90.

41. Ukena C, Kindermann M, Mahfoud F, Geisel J, Lepper PM, Kandolf R, Böhm M, Kindermann I. Diagnostic and prognostic validity of different biomarkers in patients with suspected myocarditis. Clin Res Cardiol 2014;103:743-51.

42. McNamara DM, Holubkov R, Starling RC, Dec GW, Loh E, TorreAmione G, Gass A, Janosko K, Tokarczyk T, Kessler P, Mann DL,
Feldman AM. Controlled trial of intravenous immune globulin in recent-onset dilated cardiomyopathy. Circulation 2001;103:2254-59.

43. Saegusa S, Fei Y, Takashi T, Sumino H, Moriya J, Kawaura K, Yamakawa J, Itoh T, Morimoto S, Nakahashi T, Iwai K, Matsumoto M, Kanda T. Oral administration of candesartan improves the survival of mice with viral myocarditis through modification of cardiac adiponectin expression. Cardiovasc Drug Ther 2007;21:155-60.

44. Bahk TJ, Daniels MD, Leon JS, Wang K, Engman DM. Comparison of angiotensin converting enzyme inhibition and angiotensin II receptor blocade for prevention of experimental autoimmune myocarditis. Int J Cardiol 2008;125:85-93.

45. Xiao J, Shimada M, Liu W, Hu D, Matsumori A. Antiinflammatory ef fect of eplerenone on viral myocarditis. Eur J Heart Fail 2009;11:34953.

46. Pauschinger M, Rutschow S, Chandrasekharan K, Westermann D, Weitz A, Peter Schwimmbeck L, Zeichhardt H, Poller W, Noutsias M, Li J, Schultheiss HP,Tschope C. Carvedilol improves left ventricular function in murine coxsackievirus induced acute myocarditis association with reduced myocardial interleukin-1 beta and MMP-8 expression and a modulated immune response. Eur J Heart Fail 2005;7:444-52.

47. Stanton C, Mookadam F, Cha S, McNamara D, Aukrust P, Wojnicz R, Bailey KR, Cooper LT. Greater symptom duration predicts response to immunomodulatory therapy in dilated cardiomyopathy. Int J Cardiol 2008; 128:38-41.

48. Frustaci A, Chimenti C, Calabrese F, Pieroni M, Thiene G, Maseri A. Immunosuppressive therapy for active lymphocytic myocarditis. Virological and immunologic profile of responders versus nonresponders. Circulation 2003;107:857-86. 